LBL - 36135

$\mathrm{UC}-413$

UPR - 0631T

\title{
Stationary Neutrino Radiation Transport by Maximum Entropy Closure ${ }^{\dagger}$
}

\author{
S.A. Bludman ${ }^{\ddagger}$ J. Cernohorsky ${ }^{\S}$ \\ Nuclear Science Division \\ Lawrence Berkeley Laboratory \\ University of California \\ Berkeley, California 94720, U.S.A.
}

November 23, 1994

\footnotetext{
${ }^{\dagger}$ This work was supported by the Director, Office of Energy Research, Office of High Energy and Nuclear Physics, Division of Nuclear Physics, of the U.S. Department of Energy under Contract DE-AC03-76SF00098 and by the Department of Energy contract AC02-76-ERO-3071 at the University of Pennsylvania.

${ }^{\ddagger}$ Center for Particle Astrophysics, University of California, Berkeley, California 94720 and Department of Physics, University of Pennsylvania, Philadelphia, PA 19104 U.S.A.

${ }^{\S}$ On leave from the Max-Planck Institut für Astrophysik, 85748 Garching, Federal Republic of Germany
}

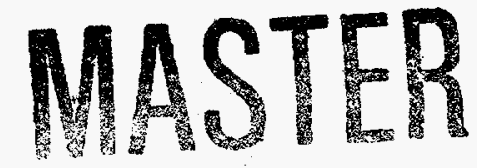




\section{DISCLAIMER}

This report was prepared as an account of work sponsored by an agency of the United States Government. Neither the United States Government nor any agency thereof, nor any of their employees, make any warranty, express or implied, or assumes any legal liability or responsibility for the accuracy, completeness, or usefulness of any information, apparatus, product, or process disclosed, or represents that its use would not infringe privately owned rights. Reference herein to any specific commercial product, process, or service by trade name, trademark, manufacturer, or otherwise does not necessarily constitute or imply its endorsement, recommendation, or favoring by the United States Government or any agency thereof. The views and opinions of authors expressed herein do not necessarily state or reflect those of the United States Government or any agency thereof. 


\section{DISCLAIMER}

Portions of this document may be illegible in electronic image products. Images are produced from the best available original document. 


\title{
StationaRy NEUTRINo RADIATION TRANSPORT BY MAXIMUM ENTROPY Closure
}

\author{
S.A. Bludman \\ Center for Particle Astrophysics and Lawrence Berkeley Laboratory, Berkeley CA 94720, \\ Department of Physics, University of Pennsylvania, Philadelphia, PA 19104

\section{J. Cernohorsky} \\ Lawrence Berkeley Laboratory, University of California, Berkeley CA 94720, U.S.A. \\ On leave from the Max-Planck Institut für Astrophysik, 85748 Garching, Federal \\ Republic of Germany
}

August 30, 1994

\begin{abstract}
We obtain the angular distributions that maximize the entropy functional for Maxwell-Boltzmann (classical), Bose-Einstein, and Fermi-Dirac radiation. In the low and high occupancy limits, the maximum entropy closure is bounded by previously known variable Eddington factors that depend only on the flux. For intermediate occupancy, the maximum entropy closure depends on both the occupation density and the flux. The Fermi-Dirac maximum entropy variable Eddington factor shows a scale invariance, which leads to a simple, exact analytic closure for fermions. This two-dimensional variable Eddington factor gives results that agree well with exact (Monte Carlo) neutrino transport calculations out of a collapse residue during early phases of hydrostatic neutron star formation.
\end{abstract}




\section{Variable Eddington Factors to Close the Hierarchy of Angular Moment Equations}

The maximal information on any radiation field is contained in the distribution function which solves the Boltzmann transport equation as function of space-time and fourmomentum. The collision integral describing the radiation-matter interaction also includes stimulated emission or absorption factors expressing the Bose or Fermi statistics of the radiation. The geometry of the problem enters through the radiation advection terms and the boundary conditions.

Exactly solving the Boltzmann equation is computationally expensive and often provides more information than is practically required. Instead, in an Eddington moment approach the hierarchy of its angular moments is often considered, at each order containing angular moments of the distribution function of at least one order higher. Therefore, an additional relation between the Eddington factors is required to close the set.

Ideally, the first two moments of the Boltzmann equation are considered, taking account of the conservation of radiation energy and momentum, and containing the first three moments of the distribution function : occupation density, flux, and pressure.

The first and second Eddington factors are defined as $f=F / e$ and $\overrightarrow{\mathbf{p}}=\overrightarrow{\mathbf{P}} / e:$ the radiation flux and the pressure normalised by the occupation density $e$, the zeroth Eddington factor. The hierarchy of moment equations may be closed with the first two moments when the second (or variable) Eddington factor, $\vec{p}=\vec{p}(\mathbf{f}, e)$ is specified as function of the flux $f$ and occupation density $e$.

The Eddington factors $\mathbf{f}$ and $\overrightarrow{\mathbf{p}}$ are normalised moments of a non-negative weight function on the unit sphere and as such obey the Schwarz inequality $f^{2} \leq p \leq 1$, with $f \equiv|\mathbf{f}|$ and $p \equiv|\overrightarrow{\mathbf{p}} \cdot \mathbf{f} / f|$. In the diffusive (isotropic) limit $f=0, p=1 / 3$. In the ballistic (free-streaming) limit, the upper equality $f=p=1$ applies.

The second angular moment of the Boltzmann equation, the momentum conservation equation, may be written as

$$
\left(p-f^{2}\right) \nabla e=-e \mathbf{f} \kappa_{\text {tot }}(e, f, p),
$$

where $\kappa_{\text {tot }}(e, f, p)$ is the total inverse mean free path, including the artificial opacity (Cernohorsky and van Weert 1992; Dgani and Janka 1992).

It defines the effective Knudsen number $R$, the quotient of the mean free path and the 
energy scale height, when rewritten as

$$
R \equiv(-\nabla \ln e) / \kappa_{t o t}=\mathbf{f} /\left(p-f^{2}\right)
$$

The effective Knudsen number is small $(R \ll 1)$ in the diffusive limit, and large $(R \gg 1)$ in the free-streaming limit. In the optically dense region the flux $F \approx-\nabla e /\left(3 \kappa_{t o t}\right)$, in the optically thin regime $\mathbf{F} \approx e$.

Many closures or variable Eddington factors have been proposed (Janka 1991; Janka 1992; Janka, Dgani and van den Horn 1992; Levermore 1984; Minerbo 1978; Pomraning 1981). All of these closures are one-dimensional, meaning that $\vec{p}$ is a function of only one of the lower order Eddington factors, $e$ or $\mathbf{f}$. While computationally efficient, these one-dimensional closures agree poorly with the results of exact Monte Carlo solutions of the neutrino transport equation (Janka, Dgani and van den Horn 1992).

The maximum entropy closure (MEC), which we define in the next section, accounts for the radiation quantum statistics by its dependence on the occupation number density $e$, in addition to $f$. This makes MEC two-dimensional, including earlier one-dimensional closures as limiting cases. We consider Bose-Einstein (BE), Fermi-Dirac (FD) and classical (Maxwell-Boltzmann, MB) radiation in a unified way and obtain formal symmetries among them. For Bose and for Fermi statistics, we find complementary two-dimensional Eddington surfaces, $p=p(e, f)$, which overlap only in the classical limit of low occupation number density.

In the next section, we derive the common algorithm used to obtain the three different maximum entropy closures. In sections 3 and 4, we discuss the classical limit and the BoseEinstein closures (MEC-BE). In Section 5, we consider the Fermi-Dirac closure (MECFD), with emphasis on limiting behaviour and symmetries. These symmetries inspire the introduction of relative variables, in terms of which MEC-FD exhibits universal scaling which leads to a simple and exact analytic expression for the closure.

Not surprisingly, our two-dimensional closures approximate radiation transport better than does any one-dimensional closure. In the concluding sections, we find indeed that MEC-FD agrees well with exact Monte Carlo transport calculations for the range of occupation densities and fluxes expected during the early stages of neutron star formation and in typical material environments after nuclear bounce in type II supernovae.

We restrict ourselves to systems with only one preferred spatial direction, relative to which vectors and tensors are defined. This includes spherically symmetric and rectangular 
geometries and permits us to treat only two independent scalars: the magnitude of the first Eddington factor $f= \pm|\mathbf{f}|$ and the forward component of the second Eddington factor, $p \equiv|\overrightarrow{\mathbf{p}} \cdot \mathbf{f} / f|$. We work within the spectral approach, in which all Eddington factors $e, f, p$ depend on the particle energy, which we suppress in the notation.

\section{Maximum Entropy Closure}

The maximum entropy method finds, in the sense of information theory, the least biased distribution based on knowing only radiation statistics, occupation density $e$ and flux $f$. The maximum entropy distribution $\psi$ is found by maximising the entropy functional

$$
s[\psi] \propto(1-k \psi) \ln (1-k \psi)+k \psi \ln \psi
$$

with $k=1$ for Fermi-Dirac , $k=-1$ for Bose-Einstein and

$$
s[\psi] \propto \psi \ln \psi
$$

for Boltzmann statistics, under the constraints that the zeroth and first angular moment of the distribution function

$$
\begin{aligned}
& e=\frac{1}{4 \pi} \int_{0}^{2 \pi} d \phi \int_{-1}^{1} \psi d \mu \\
& f=\frac{1}{4 \pi e} \int_{0}^{2 \pi} d \phi \int_{-1}^{1} \mu \psi d \mu
\end{aligned}
$$

equal the prescribed number density or phase space occupancy $e$ and the normalised flux $f$. The quantity $\mu=\cos \vartheta$ is the cosine of the normalised direction of the particle momentum with respect to the preferential spatial axis. The normalised forward pressure is

$$
p=\frac{1}{4 \pi e} \int_{0}^{2 \pi} d \phi \int_{-1}^{1} \mu^{2} \psi d \mu
$$

Following a standard procedure from the calculus of variations (Minerbo 1978), the constraints (2.3) and (2.4) introduce Lagrange multipliers $\eta, a$ and after some rewriting we obtain the general form of the maximum entropy distribution function

$$
\psi(\mu)=\frac{1}{e^{a(z-\mu)}+k}=\frac{1}{B e^{-a \mu}+k}
$$


Here $a$ is the inverse angular "temperature", $z \equiv \eta / a$ the inverse angular "chemical potential", $B \equiv \exp \eta$ the reciprocal fugacity, $k=1,-1,0$. For outwardly directed radiation $f \geq 0$, so that $a \geq 0$. (Because $f(\eta,-a)=-f(\eta, a)$, the treatment of backward-peaked radiation is straightforward.) For Bose-Einstein and Maxwell-Boltzmann $(k=-1,0)$ statistics, the occupancy $0 \leq e \leq \infty$ is unrestricted and $1 \leq z \leq \infty$. For Fermi-Dirac statistics $(k=1)$, the occupancy $0 \leq e \leq 1$ and $z$ is unrestricted.

\subsection{Limiting Cases or Approximations Give One-dimensional Closures}

Both quantum statistics reduce to the classical (Boltzmann) limit when the occupancy $e \ll 1$. The opposite limit is reached for $e \rightarrow \infty$ (high occupancy) in the Bose-Einstein case, and for $a \rightarrow \infty$ (maximum packing) in the Fermi-Dirac case. These three limits lead to the angular distributions and one-dimensional Eddington factors in Table 1.

Table 1: Limiting angular distribution functions and maximum entropy closures $p(f)$ for angular distribution functions $\psi(\mu)=[\exp a(z-\mu)+k]^{-1}$ of three statistics. The function $q(L)$ is the inverse of the Langevin function $L(q) \equiv \operatorname{coth} q-1 / q$.

\begin{tabular}{l|c||cc|cc}
\hline Statistics & $\mathrm{k}$ & $\psi(\mu)$ & $\mathrm{p}(\mathrm{f})$ & Closure \\
\hline \hline $\mathrm{BE}$ & -1 & $1 / a(z-\mu)$, & $e \gg 1$ & $f \operatorname{coth} q(f)$ & Levermore-Pomraning \\
\hline $\mathrm{MB}$ & 0 & $\exp a(\mu-z)$, & $e \ll 1$ & $1-2 f / q(f)$ & Minerbo \\
\hline $\mathrm{FD}$ & 1 & $\theta(\mu-z)$, & $a \rightarrow \infty$ & $\left(1-2 f+4 f^{2}\right) / 3$ & Maximal Packing \\
\hline \hline
\end{tabular}

The two constraints make the Lagrange multipliers $\eta$ and $a$ implicit functions of the number density $e$ and flux $f$. To arrive at a closure $p=p(e, f)$, we need to obtain $\eta(e, f)$ and $a(e, f)$ by inversion of the constraints $(2.3,2.4)$. To facilitate this inversion, previous authors approximated the maximum entropy distribution function and obtained various one-dimensional closures: Minerbo (1978) considered the maximum entropy distribution in the classical limit ( $e \ll 1$ )and found an analytic, but implicit, closure. In the Bose case, Pomraning (1981) and in the Fermi case, Cernohorsky, van den Horn and Cooperstein (1989) approximated (2.6), to obtain an explicit analytic inversion. Both these latter approximations lead to the one-dimensional Levermore-Pomraning Eddington factor. 


\subsection{Eliminating the Constraints Gives Two-dimensional Closures}

We now formulate the closure without approximating the angular distribution (2.6). The differential identity for $k= \pm 1$,

$$
\psi \equiv \frac{k}{a} \frac{\partial g(\mu)}{\partial \mu} \quad, g(\mu) \equiv-\ln (1-k \psi)=\ln [1+k \exp (a(\mu-z)]
$$

allows integrating by parts

$$
\int_{-1}^{1} \mu^{n} \psi d \mu=\frac{k}{a}\left[\left\{\mu^{n} g(\mu)\right\}_{-1}^{1}-n \int_{-1}^{1} \mu^{n-1} g(\mu) d \mu\right] .
$$

The first constraint with $n=0$ now follows without integration and can be inverted to express the reciprocal fugacity

$$
\exp \eta \equiv B(e, a)=\frac{\sinh (1-k e) a}{\sinh (e a)}
$$

as a function of $e$ and $a$. This quantity has two useful symmetries:

$$
\begin{aligned}
B(e, a) & =B(e,-a) \\
B^{-1}(e, a) & =B(k-e, a) .
\end{aligned}
$$

The first symmetry ensures backward-forward symmetry $f(e,-a)=-f(e, a)$. The second symmetry has no physical consequences, in the Bose-Einstein case. But in the FermiDirac case, $e$ and $1-e$ are both positive, so that the second symmetry relates occupied to unoccupied states. In section 5 , these symmetries point the way to an analytic form for the MEC-FD variable Eddington factor $p=p(f, e)$.

With $\eta$ as a function of $e$ and $a$, one Lagrange multiplier is eliminated from the remaining two Eddington factors $f$ and $p$. We use equation (2.8) with $n=1$ to eliminate the second Lagrange multiplier $a$ by numerically inverting the second constraint (2.4). The remaining integral is numerically more tractable than the original one in equation (2.4), and is solved with a Gauss-Legendre quadrature. For given $e$ and $f$, the inversion of constraint (2.4) is now a one-dimensional root-finding problem, which we solve by a combination of a False Position and Newton-Raphson algorithm.

Having inverted the constraint (2.4) to obtain $a=a(e, f)$, equation (2.8) with $n=2$ now yields $p(e, f)$. With this procedure, we have a numerical algorithm that inputs $e, f$, and returns the two-dimensional variable Eddington factor $p(e, f)$. But in the Fermi-Dirac case that interests us, we will obtain an analytic form for the variable Eddington factor! 


\section{Maximum Entropy Closure in the Classical Limit}

With either Bose or Fermi statistics, when the occupation $e \ll 1, B \approx \sinh a / e a$ becomes very large, and the quantum index $k$ in the denominator of $\psi$ can be neglected. With $k=0$, the integrals $(2.4,2.5)$ can be performed analytically

$$
f(a)=L(a), \quad p(a)=1-2 f(a) / a
$$

where $L(q) \equiv \operatorname{coth} q-1 / q$ is the Langevin function, running monotonically from $q / 3$ to 1 , and $\mathrm{q}(\mathrm{L})$ is its inverse. This leads to Minerbo's one-dimensional closure $p=p(f)=$ $1-2 f / q(f)$, the limiting curve that separates MEC-BE and MEC-FD.

\section{Maximum Entropy Closure for Bose-Einstein Radiation}

For small occupation density $(e \ll 1)$, the distribution tends to the Boltzmann limit and the variable Eddington factor tends to the Minerbo form discussed in the preceding Section. For large occupation density ( $e \gg 1$ ), the denominator of $\psi(\mu)$ is small and can be expanded to give

$$
\psi(\mu) \approx \frac{1}{a(z-\mu)}, e \gg 1
$$

In the constraints $(2.3,2.4)$ and in equation $(2.5)$, the angular integrals may be performed analytically leading to

$$
p=z f(z) \quad, \quad f(z)=z-2\left[\log \left(\frac{z+1}{z-1}\right)\right]^{-1}=\operatorname{coth} q-1 / q \equiv L(q)
$$

where $\operatorname{coth} q \equiv z$. Thus,

$$
p=f \operatorname{coth} q(f)=f\left(f+q^{-1}\right),
$$

so that $q$ is recognized as the Knudsen number $R$ in equation (1.2). This limiting trajectory, the 'logarithmic limit' discussed by Fu (1987), is none other than the LevermorePomraning closure (Levermore and Pomraning 1981). Because the Levermore-Pomraning and Minerbo closures express the flux as the Langevin function of different variables $q$ and $a$, the MEC-BE and MEC-MB variable Eddington factors summarized in Table 1 are different.

For intermediate values of $e$ and $f$, we find the maximum entropy distributions $\psi(e, f)$, and the variable Eddington factor $p=p(f, e)$ by the numerical inversion described in Section 2. 
Figure 1 shows the MEC-BE surface $p=p(f, e)$, parametrised by the occupancy $e$, and bounded by the Minerbo Eddington trajectory for $e \ll 1$ and by the Levermore-Pomraning trajectory for $e \gg 1$.

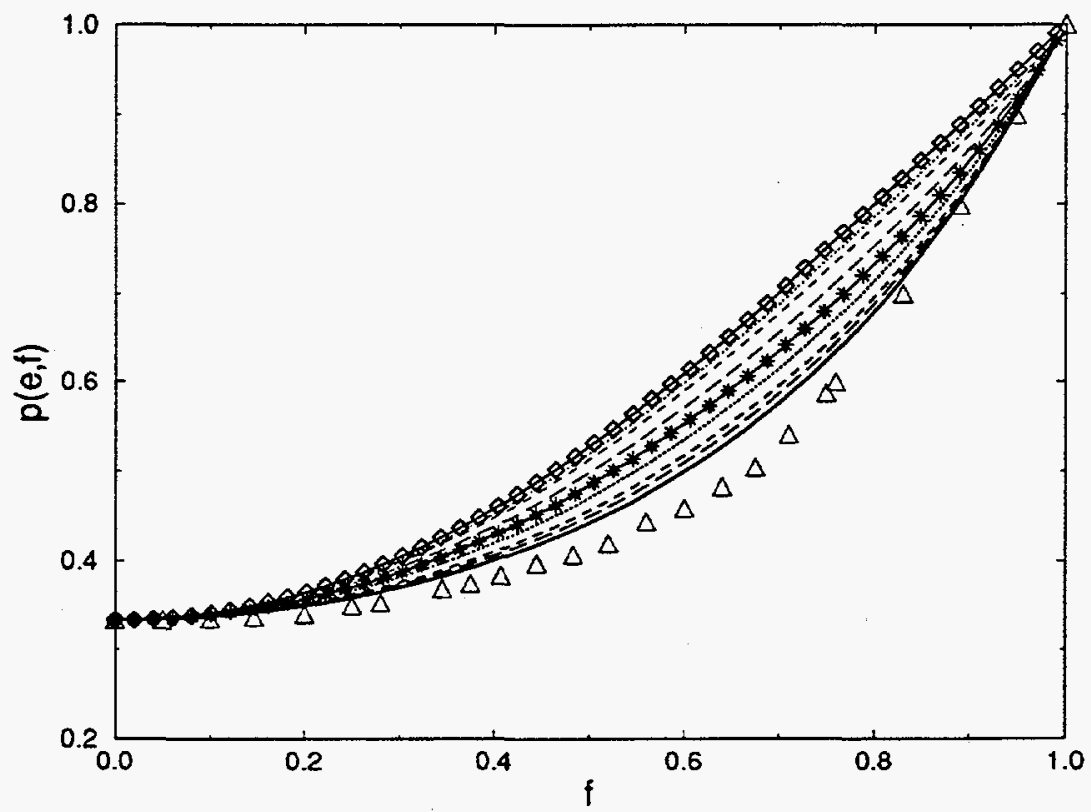

Figure 1: Maximum entropy Bose-Einstein closure for nine occupancies bottom-to-top $e=0,0.1,0.2,0.5,1 .(*), 2 ., 5 ., 10, \infty(\diamond)$. Bose-Einstein closure allows a family of trajectories between the Minerbo trajectory $p(f)=1-2 f / q(f)$ (bottom-) and the Levermore-Pomraning trajectory $p(f)=f \operatorname{coth} q(f)$ (top $\diamond)$. The exact energy-averaged neutrino trajectory obtained by Janka $(\triangle)$ lies below the range of MEC-BE.

\section{Maximum Entropy Closure for Fermi-Dirac Radiation}

Equation (2.9) implies that in the limit $a \rightarrow \infty, z \rightarrow(1-2 e)$, so that the angular distribution function approaches a step-function with Fermi surface at $\mu=z$. In this limit, called maximal packing (Janka, Dgani and van den Horn 1992), the integrals (2.4, 2.5) can be done analytically, giving $f_{\max }=1-e, p_{\max }=1-2 e+(4 / 3) e^{2}$. Figure 3 shows the maximum entropy Eddington surface $p(f, e)$ labeled by $e$ and bounded by the Minerbo trajectory and the maximal packing curve $p=\left(1-2 f+4 f^{2}\right) / 3$.

A remarkable scaling law appears for arbitrary degeneracy, when we define relative 


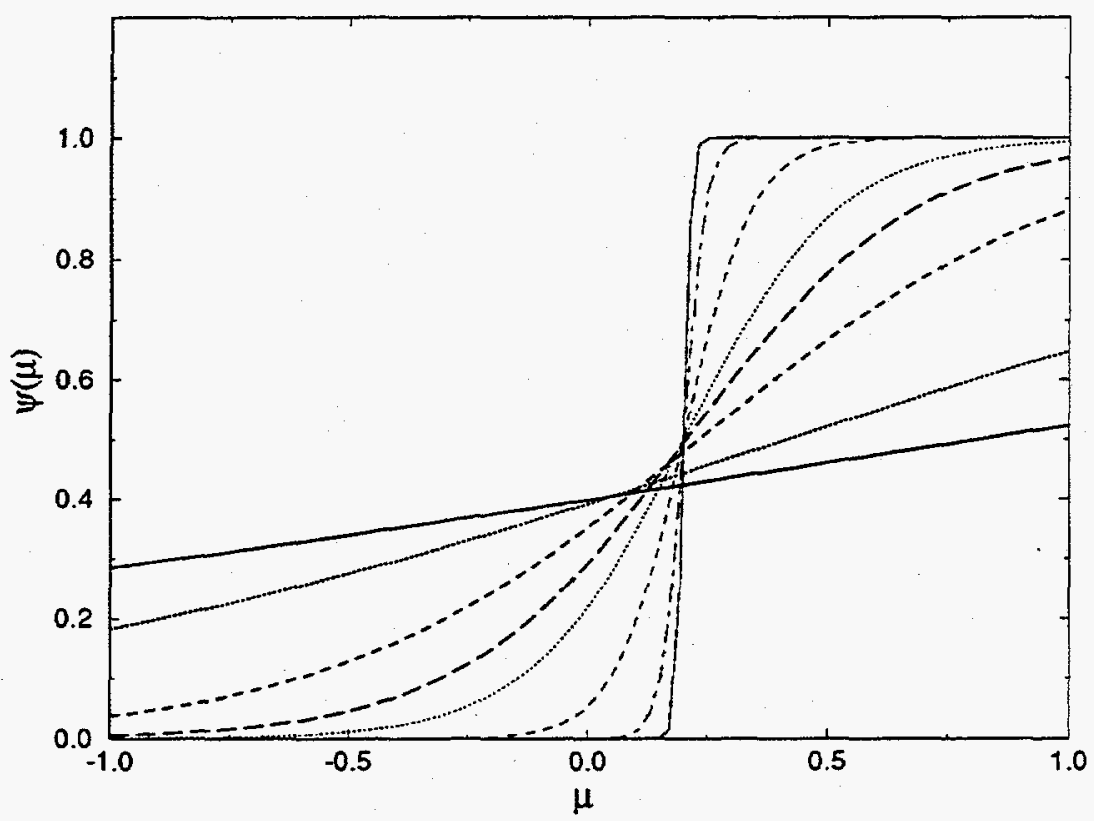

Figure 2: Maximum entropy FD distribution $\psi(\mu)$ for occupancy $e=0.4$. and the allowed fluxes $=0.1(--), 0.2,0.4,0.5,0.55,0.59,0.599,0.5999(-)$. The sharpness of the Fermi surface at $\mu=z=1-2 e$ increases with the flux.

coordinates

$$
x(e, a) \equiv \frac{f(e, a)}{f_{\max }(e)} \quad, \quad \chi(e, a) \equiv \frac{p(e, a)-1 / 3}{p_{\max }(e)-1 / 3},
$$

called 'flux-saturation' and 'pressure-saturation'. These relative coordinates are normalized to run from zero to one as a goes from zero to infinity and the radiation field passes from isotropic to maximally packed. The symmetry (2.11) makes these relative coordinates invariant under the 'particle-hole exchange' $e \rightarrow 1-e$, at fixed $a:$

$$
x(e, a)=x(1-e, a) \quad, \quad \chi(e, a)=\chi(1-e, a)
$$

After the Lagrange multiplier $a$ is eliminated, how is the particle-hole $e \rightarrow 1-e$ symmetry expressed in the variable Eddington factor $p(e, f)$ ? With $a$ eliminated numerically in equation (5.1) to express the variable pressure-saturation

$$
\chi(e, x) \equiv \frac{p\left(e, x f_{\max }(e)\right)-1 / 3}{p_{\max }(e)-1 / 3},
$$

as function of the flux saturation $x$, we find that $\chi(e, x)$ is not only invariant under $e \rightarrow 1-e$, but entirely independent of $e$, a universal function of $x$ only, as shown by the solid curve in figure 4. Since $\chi$ is independent of $e$, we may insert into equation (5.3) the classical 


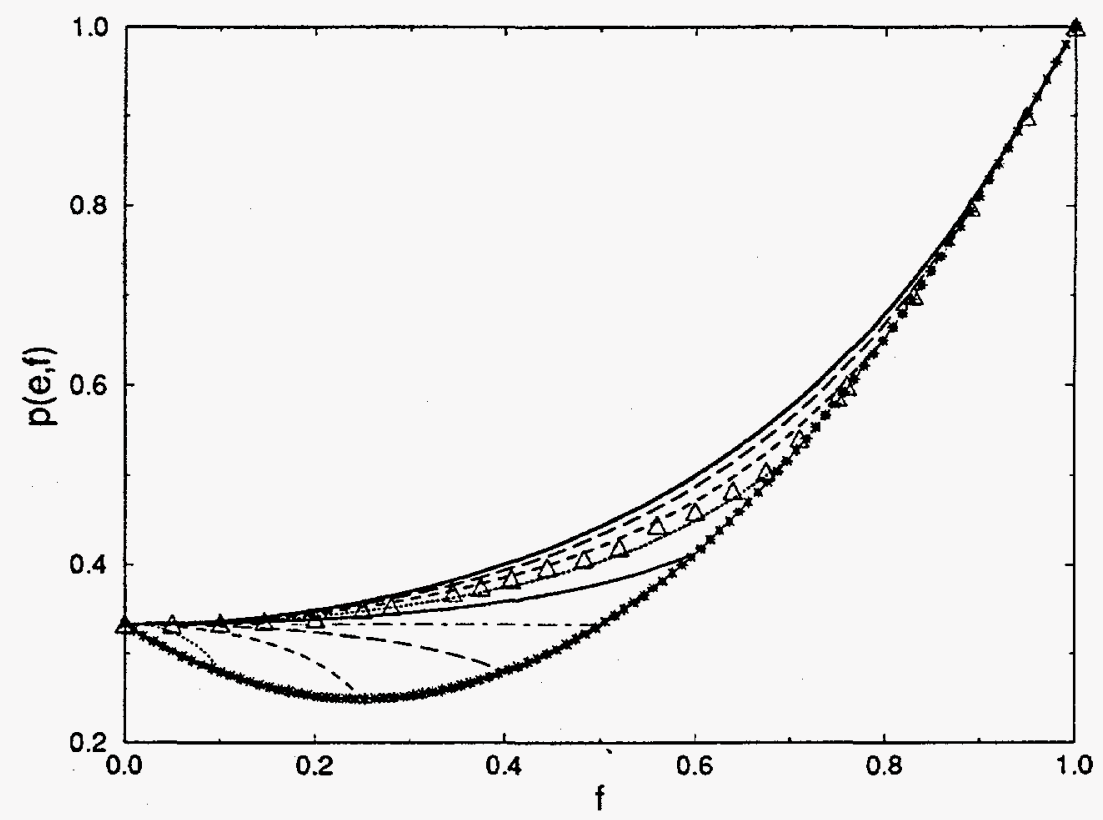

Figure 3: MEC-FD surface $p(e, f)$. The uppermost curve (-) is the classical (Minerbo) limit $p(f)=1-2 f / q(f)$. The bottom curve $((* \cdots *)$ is the maximal packing envelope $p(f)=\left(1-2 f+4 f^{2}\right) / 3$. Between these lie $p(f, \epsilon)$ with $e=0.1,0.2,0.3,0.4,0.5(---)$, $0.6,0.75,0.9$ from top to bottom. The energy-averaged Eddington factors from Janka's Monte Carlo calculations are marked by $(\triangle)$ and all lie below the classical limit.

limit (3.1), obtaining exactly

$$
\chi(x)=1-3 x / q(x)
$$

where $q(x)$ is the inverse Langevin function. This curve matches the numerical curve in figure 4 , and may be approximated to $2 \%$ accuracy by

$$
\chi(x)=x^{2}\left(3-x+3 x^{2}\right) / 5
$$

the lowest order polynomial with exactly the correct limiting behaviour. This polynomial approximation is shown by the long-dashed curve in figure 4 .

From equation (5.3), the variable Eddington factor

$$
p(e, f)=\frac{2(1-e)(1-2 e)}{3} \chi\left(\frac{f}{1-e}\right)+\frac{1}{3},
$$

is thus expressed in terms of the universal pressure saturation (5.4). Either the exact expression (5.4) or its approximation (5.5) reproduce the variable Eddington factor surface shown in Fig. 3. The relative error of the polynomial approximation is, at worst, 
of the order of a few per thousand. The laborious numerical inversion needed for the Bose-Einstein Eddington factors can, in the Fermi-Dirac case, be substituted by a 10-line algorithm!

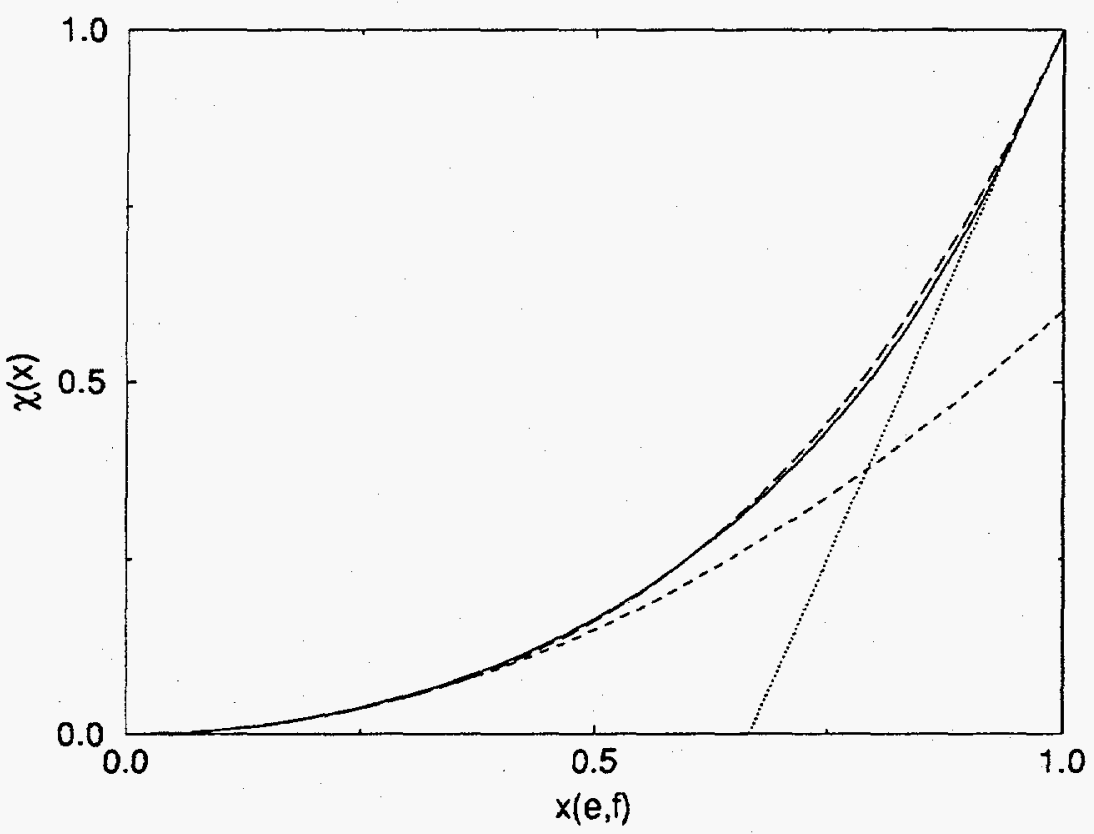

Figure 4: The universal variable pressure-saturation $\chi(x)$, equation (5.4) as a function of the flux-saturation $x$. The dotted line is the free-streaming limit $\chi(x \rightarrow 1) \approx 3 x-2$, the dashed line is the diffusive limit $\chi(x \rightarrow 0) \approx 3 x^{2} / 5$. The long-dashed curve is the polynomial fit, equation (5.5).

\section{Comparison with Monte Carlo Eddington Factors}

In this section, we compare our MEC variable Eddington factors with variable Eddington factors calculated from exact Monte Carlo solutions of the neutrino transport equation, for a variety of material backgrounds, during the early neutrino-cooling phase of neutron star formation shortly after shock-launch (Dgani and Janka 1992; Janka 1991; Janka 1992; Janka, Dgani and van den Horn 1992). These Eddington factors are energy-averaged and fall in a narrow bound about $e=0.25-0.3$ for $f<0.7$ and near the maximal packing envelope for $f>0.7$, within the MEC-FD region in Fig. 3 and entirely outside the MEC$\mathrm{BE}$ region in Fig. 1. 


\subsection{Neutrino Cooling Proto Neutron Star After Bounce}

At least two factors contribute to this remarkably universal behaviour of the Monte Carlo Eddington factors. Firstly, the Monte Carlo calculations were performed in semi-transparent regions of stellar models, several seconds after bounce, during the early phases of the hydrostatic neutron star formation, when the delayed explosion mechanism presumably operates. The shock wave has by then traversed the semi-transparent region, dissociated the heavy nuclei present, and left a mixture of nucleons and $\alpha$ 's. The chemical compositions and the matter density profiles determining the neutrino opacity must therefore be similar in all models. This largely explains the model-to-model universality.

Secondly, while the Monte Carlo spectral Eddington factors would scatter in the $p-f$ plane, they must lie within the MEC-FD surface. For this evolutionary epoch and stellar environment, the main contribution to the energy average apparently comes from bins with moderate occupation density, $e(\omega) \sim 0.2-0.3$ and moderate flux. At higher fluxes $f>0.7$, bins with even smaller occupation density $e<0.1$ dominate. This explains why the Minerbo (Boltzmann) closure, often works fairly well for a range of Monte Carlo Eddington trajectories.

Under unusual conditions, when narrow shell sources in the semi-transparent region occur together with a steep gradient in opacity, so that large occupation densities coincide with high fluxes, Janka reports $p<1 / 3$. In such special circumstances, the Monte Carlo results could not be described by any one-dimensional closure or direct phenomenological fit to the Monte Carlo data such as (Dgani and Janka 1992; Janka 1991), but would be well approximated by MEC-FD with $e>1 / 2, f \rightarrow f_{\max }$.

Comparison with other figures in Janka (1991), Janka (1992), Janka, Dgani and van den Horn (1992) of Monte Carlo energy-integrated local neutrino angular distributions shows that the MEC-FD distribution in figure 2 describes the exact distribution, at least qualitatively. Because degenerate fermion transport is dominated by the exclusion principle, the MEC-FD statistical treatment captures the most essential transport feature.

\subsection{Stationary Neutrino Transport Out of a Shocked Supernova Core}

We have incorporated MEC-FD into the transport code and tested it in a transport calculation on a stationary model $1.5 \mathrm{M}_{\odot}$ stellar core. The stellar background used is model M0 from Cernohorsky and van Weert (1992), described there in greater detail. 

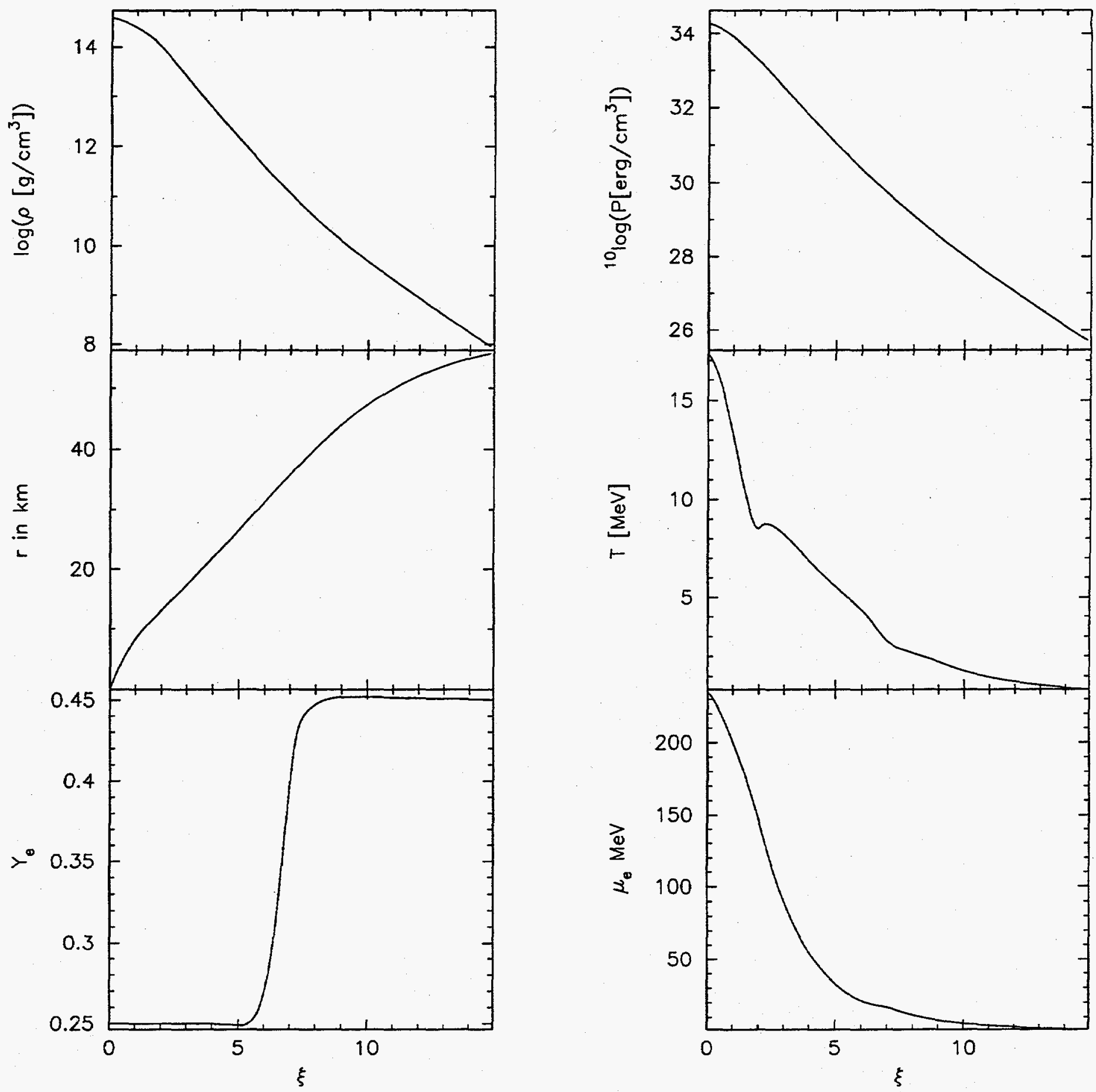

Figure 5: Matter background as function of Lagrangean position, clockwise from upper left: density, pressure, temperature, electron chemical potential, electron concentration (per nucleon) $Y_{e}=Y_{p}=1-Y_{n}$, radial position $(\mathrm{km})$ 
It is a tri-polytrope put in hydrostatic equilibrium in Newtonian gravity without including the neutrino component, and subsequently evolved for $10^{-5} \mathrm{sec}$, after switching on the neutrinos. The chemical composition is an ideal gas of totally dissociated matter containing nucleons, photons and an electron-positron mixture. Above nuclear matter densities a hard, Skyrme-type term to the pressure is added (Cernohorsky 1990). The density, temperature, pressure and composition profiles for this material background are depicted in figure 5 as function of the Lagrangean variable

$$
\xi=\ln \left(1-\left[\frac{m_{r}}{M_{\star}(1+\epsilon)}\right]^{1 / 3}\right)
$$

to give spatial resolution in the outer regions containing little mass.

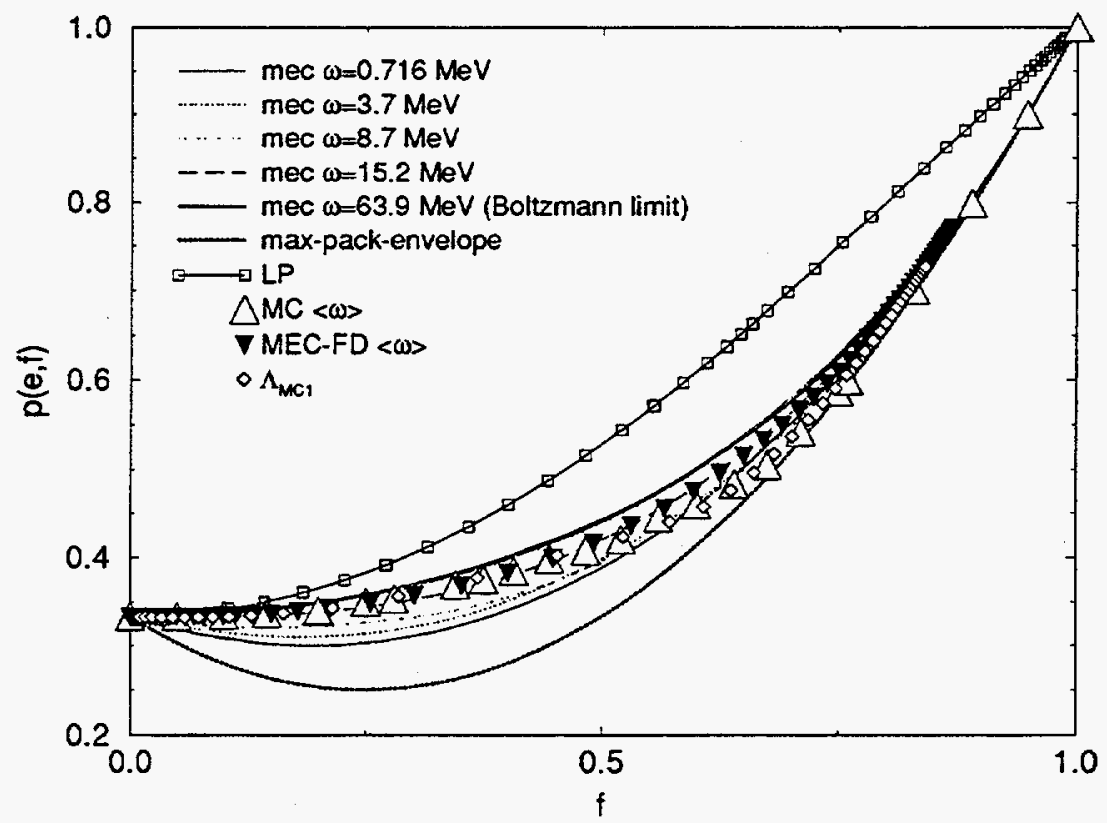

Figure 6: Eddington trajectories from top to bottom : Levermore-Pomraning (LP) (ם), spectral MEC-FD for all energy bins, from high to low energies, the high energy bins clustering on the Boltzmann limit, maximal packing envelope. Energy average of MECFD $(\nabla)$, Monte Carlo $(\mathrm{MC})$ trajectory $(\triangle)$, MC-fit closure $\Lambda_{M C 1}(\diamond)$

Keeping the stellar background fixed in time, we calculate the stationary solutions of the first two moments of the Boltzmann equation, the energy balance and momentum balance equation, using different closures including MEC-FD. We start from some initial guess for $e, f$ and $p$ and let the transport equations evolve until a stationary state is reached. The calculations are spectral and monochromatic, with 14 energy bins between 


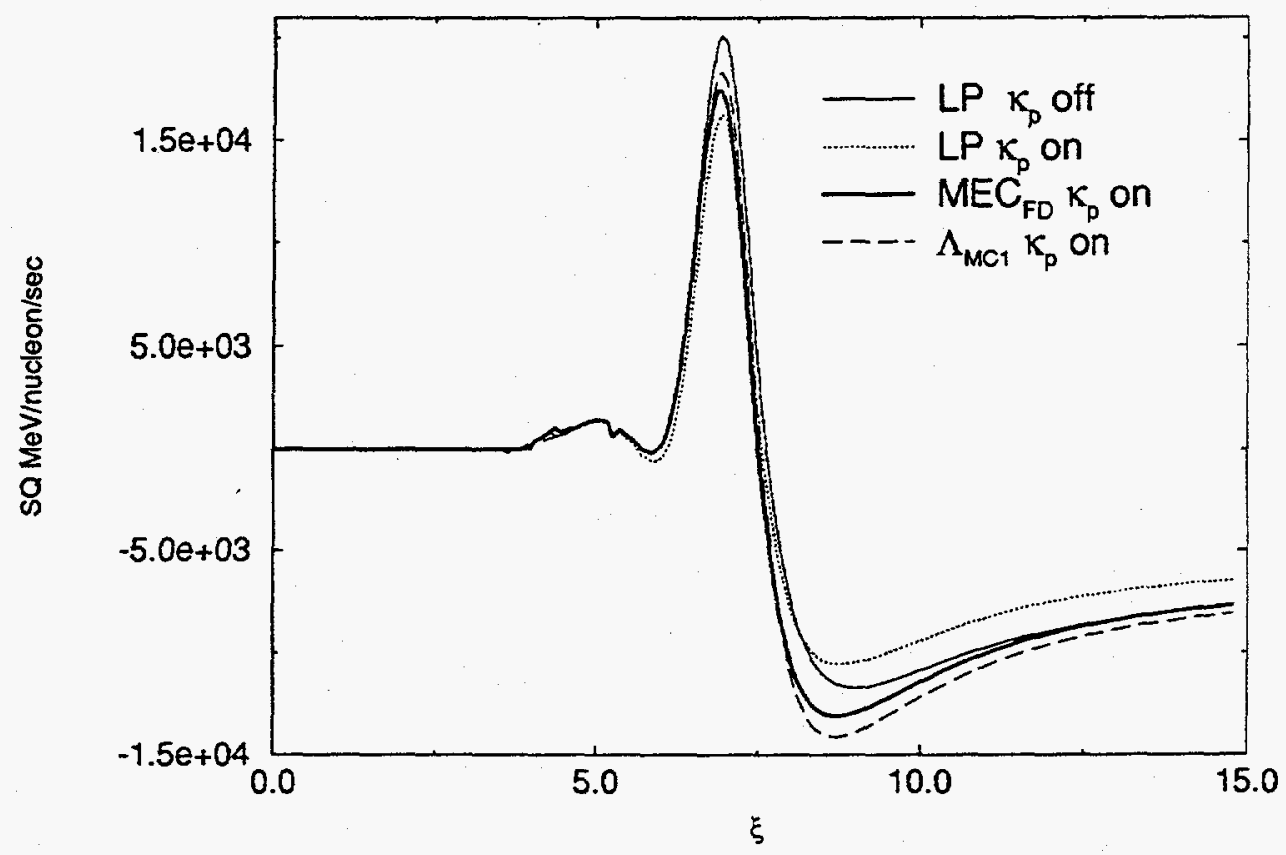

Figure 7: Total ( $\omega$-integrated) matter-neutrino energy transfer as function of position, in $\mathrm{MeV} /$ nucleon/sec for all closures. From top to bottom near $\xi=9:$ LP, LP-FLD, MEC-FD, $\Lambda_{M C 1}$.

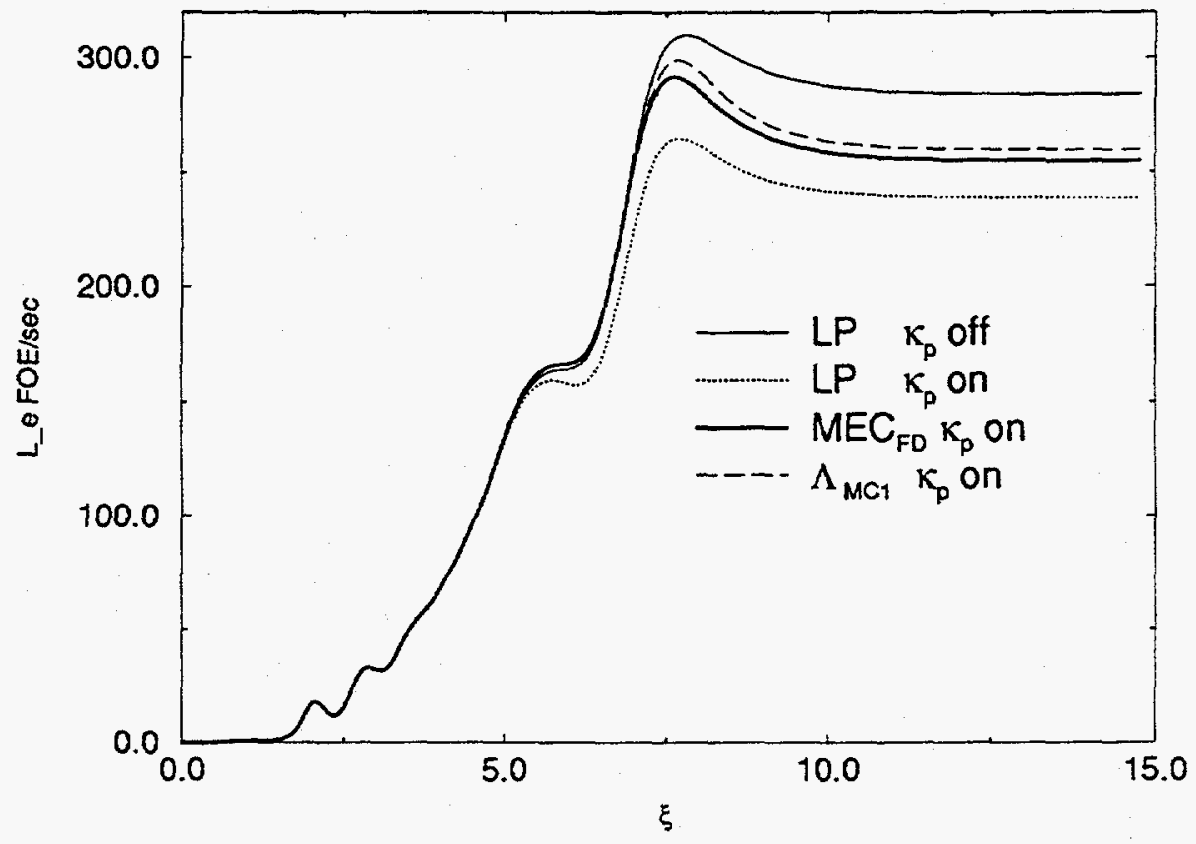

Figure 8: Total ( $\omega$-integrated) neutrino energy luminosity as function of position, in foe $\left(10^{51} \mathrm{erg}\right) \mathrm{sec}^{-1}$ for all closures. From top to bottom near $\xi=10$ : LP-FLD, $\Lambda_{M C 1}$, MEC-FD, LP. 


\section{7 and $214 \mathrm{MeV}$.}

Figure 6 shows the scatter of the spectral MEC-FD Eddington trajectories, obtained by plotting all $(f(\xi, \omega), p(\xi, \omega))$ over the whole grid, about their energy-average (black triangle). The energy-averaged Monte Carlo (MC) trajectory (large open triangles) is plotted for reference. One must keep in mind however, that the MC calculations were not carried out on the same stellar background. Also plotted, is the Eddington trajectory using the phenomenological Monte-Carlo fit closure $\Lambda_{M C 1}$ (open diamonds) from Janka (1992). All the trajectories lie in the MEC-FD surface, between the classical and maximal packing curves, and very far below the Levermore-Pomraning (LP) curve. From the spectral MECFD trajectories it is apparent that the low energy bins are in places slightly degenerate $(p<1 / 3)$, whereas the high energy bins $(\omega>50 \mathrm{MeV})$ are nowhere highly populated, hence their trajectories lie near or on the Boltzmann limit.

With these spectral Eddington factors, we calculated the stationary $\nu_{e}$ energy transfer rate between stellar background and neutrinos (figure 7) and the energy luminosity (figure 8), for all the different closures (LP,MEC-FD, $\Lambda_{M C 1}$ ) used. The thin full line in both figures corresponds to transport using the LP closure, but with the 'momentum (artificial) opacity' $\kappa_{p}$ switched off, so that the scheme does not conserve momentum and reverts to a pure flux-limited diffusion (FLD) approach. In the dense core down to a density of $10^{13} \mathrm{~g} \mathrm{~cm}^{-3}$, the transport is diffusive and all closures agree. In semi-transparent regions with MECFD the matter cools less than with LP, $\kappa_{p}=0$ and with $\Lambda_{M C 1}$, but more than with LP, $\kappa_{p} \neq 0$. In the outer streaming regions the neutrinos deposit more energy in the matter with MEC-FD than with LP, and slightly less than with $\Lambda_{M C 1}$. The MEC-FD luminosity in the outer regions is significantly lower than in LP-FLD, slightly lower than in $\Lambda_{M C 1}$ and quite a bit higher than in LP.

\section{Conclusions}

We have used maximum entropy closure to obtain the two-dimensional variable Eddington factors $p(f, e)$ for Fermi-Dirac, Bose-Einstein, and Maxwell-Boltzmann radiation transport. These Eddington factors reduce to the one-dimensional maximal packing, Levermore-Pomraning, and Minerbo Eddington factors $p(f)$ in the limits of high and low occupancy $e$ respectively. The Levermore-Pomraning closure applies to degenerate Bose transport and therefore is in principle the worst possible one-dimensional closure 
for fermions (except in the diffusive or free-streaming regimes, where all closures agree). For Fermi-Dirac radiation, we obtained the analytic formula (5.6), but for Bose-Einstein radiation a numerical inversion algorithm is still needed.

Maximum entropy closure captures the statistical features of radiation transport away from boundaries. We therefore expect it to be a good approximation for fermions, where the exclusion principle dominates short-range correlations, but a poorer approximation for bosons, where the statistics enhances short-range correlations.

Bose-Einstein statistics enhances the forward peaking of radiation, tending towards condensation into a single angular state. In astrophysical environments, where the radiation field is far from local thermodynamic equilibrium, such as stellar atmospheres, the photon occupation density is generally low $(e \ll 1)$ so that the classical limit (Minerbo 1978) will usually be the best and most efficient statistical closure. The LevermorePomraning Eddington factor, the high occupation limit of MEC-BE, should generally give less realistic results. It remains to be seen whether MEC-BE will be as useful as MEC-FD which, for neutrino radiation, reproduces and helps explain the exact Monte Carlo radiation transport shortly after shock launch and during neutron star formation. Because the variable Eddington factor depends on occupancy $e$ as well as flux $f$, it can approximate exact neutrino radiation transport in various environments better than any one-dimensional fit or closure does. The popular Levermore-Pomraning closure in comparison underestimates $\nu_{e}$ energy exchange with matter and luminosity.

Acknowledgement This work is based on an earlier paper, to be published in Astrophysical Journal (1994), and was presented at "The Physics of Supernovae" conference at the Franklin Institute, in March, 1994. It was supported by the Director, Office of Energy Research, Office of High Energy and Nuclear Physics, Division of Nuclear Physics, of the U.S. Department of Energy under Contract DE-AC03-76SF00098 at the Lawrence Berkeley Laboratory and by the Department of Energy contract AC02-76-ERO-3071 at the University of Pennsylvania. S.A.B. thanks the Center for Particle Astrophysics and the Nuclear Science Theory Division of the Lawrence Berkeley Laboratory for hospitality during the summer of 1993. J.C. thanks the Max-Planck Institut für Astrophysik for the use of their computing resources. 


\section{References}

Balian, R.1991, From Microphysics to Macrophysics, vol. 2, Springer Verlag

Cernohorsky, J.1990, Neutrino Driven Neutron Star Formation, Univ. of Amsterdam, PhD Thesis

Cernohorsky, J., van den Horn, L. J. and Cooperstein, J. 1989, J. Quant. Spectros. Rad. Transf., 42,603

Cernohorsky, J. and van Weert, C. G. 1992, ApJ , 398, 190

Dgani, R. and Janka, H. -T. 1992, Astr. Ap. , 256, 428

Fu, A. 1987, ApJ , 323, 211

Janka, H. -T.1991, Neutrino Transport in Type II Supernovae and Protoneutron Stars by Monte

Carlo Methods, Max-Planck Institut für Astrophysik, F.R.G., PhD Thesis 1992, Astr. Ap., 256, 452

Janka, H. -T., Dgani, R. and van den Horn, L. J.1992, Astr. Ap., 265, 345

Levermore, C. D. 1984, J. Quant. Spectros. Rad. Transf. , 31, 149

Levermore, C. D. and Pomraning, G. C. 1981, ApJ , 248, 321

Minerbo, G. N.1978, J. Quant. Spectros. Rad. Transf., 20, 541

Pomraning, G. C. 1981, J. Quant. Spectros. Rad. Transf. , 26, 385 